Discussion Simprovisation is an innovative style of simulationbased education that allows learners to effectively define and address their own learning needs. Placing the learner in control of the content of the study day is the principal difference between Simprovisation and standard models of simulation. We found that medical students were able to highlight and address learning needs that may not have been fully covered through our existing portfolio of simulation scenarios. We found learners used the opportunity to build on knowledge and share it with peers.

\section{G193 CAN ONLINE LEARNING RESOURCES OVERCOME THE CHALLENGES OF DISTANCE LEARNING FOR POSTGRADUATE TRAINEES IN PAEDIATRICS WITHOUT IMPACTING PERFORMANCE?}

${ }^{1} S$ Ogden, ${ }^{2} \mathrm{~S}$ Williams, ${ }^{2} \mathrm{~K}$ Barnes, ${ }^{1} \mathrm{C}$ Morgan. ${ }^{1}$ Neonatal Unit, Liverpool Women's Hospital, Liverpool, UK; ${ }^{2}$ Kid's Health Matters, Liverpool, UK

\subsection{6/archdischild-2020-rcpch. 163}

Background We have previously demonstrated that key components of the regional PGME paediatric teaching programme can be shared with the Advanced Clinical Practice (ACP) Paediatric MSc Programme. Both share archived material online and live streamed teaching sessions. This allows students geographically distant from the teaching location to access the MSc Programme. A recent survey of our paediatric trainees returning to training (RTT) revealed a similar need for accessing the regional training programme from more distant locations. We investigated whether the same learning outcomes can be achieved with remote online access by comparing how ACP trainees access the learning resources and their summative assessment results based on their distance from the teaching location.

Methods We stratified ACP trainees according to distance between workplace and teaching location with those in the upper quartile classified as distance learners. Using student's access data, we calculated the number and length of sessions accessed online. The amount of sessions where online resources were used instead of face to face teaching was compared between the distance learning group and the remaining trainees, alongside their mean exam performance across the MSc programme.

Results 77 trainees' data was analysed with a range in distance of $0-264$ miles from the teaching location (median 8.5 miles, IQR 0-28 miles). 475 hours of access data was analysed from all 4 modules of the course over 3 years. Distance learners attended more live streamed sessions online than those who lived closer (median $84 \%$ vs $32 \%$ $\mathrm{p}=0.00001$ ) yet the amount of archived material accessed was similar $(14.4$ vs $15.4 \mathrm{hr}, \mathrm{p}=0.9442)$. This suggests it is convenience rather than a predisposition that encourages students to choose online access methods. Despite this we did not demonstrate any difference between their summative exam result $(70 \%$ vs $67 \% \quad \mathrm{p}=0.169)$ or within individual modules, years or pathways.

Conclusion ACP MSc students prefer online methods of learning if they are based a greater distance from teaching locations and this does not impact their performance in summative assessments. This indicates paediatricians RTT with similar distance learning needs may also benefit from accessing online training opportunities in this way.
G194 STEP-UP TO CONSULTANT: INNOVATIVE SIMULATION PREPARING TRAINEES FOR THE CONSULTANT ROLE

R Winter, D Magnus. Bristol Medical Simulation Centre, Bristol Royal Hospital for Children, Bristol, UK

\subsection{6/archdischild-2020-rcpch. 164}

Background The transition from paediatric trainee to consultant has long been acknowledged as challenging. The RCPCH has implemented the START assessment and 'Stepping Up' programme to address this, but senior paediatric trainees remain apprehensive about their first consultant roles. When surveyed, they express particular concern about decisions trainees rarely get to practice, such as stopping CPR, managing junior colleagues and being on call from home.

Aims Design a simulation-based education programme to prepare general and subspecialty paediatric trainees for the consultant role.

Methods We conducted thematic analysis of trainee concerns from a 2017 RCPCH conference survey. These themes were developed into a framework of six domains which described different aspects of the consultant role: leader, educator, nurturer, juggler, negotiator and communicator. We designed challenging scenarios to test these domains, relevant to all trainees, and delivered two days of simulation-based education to 11 ST6-8 candidates. Given the high degree of difficulty, we recruited a specialist consultant-heavy faculty and took steps to protect candidates' psychological safety. Our programme included simulation innovations such as waking candidates with an overnight phone call, managing multiple clinical areas simultaneously and balancing clinical and non-clinical pressures.

Results 10 of 11 candidates gave us feedback, with 10/10 reporting they felt significantly more confident and competent to perform as consultants. 9/10 candidates felt simulation was superior to all other methods in preparing for difficult aspects of the consultant role. 6 candidates later approached us independently to report that they felt our course was more useful than the RCPCH START assessment.

Conclusions We have demonstrated that simulation is an effective tool to prepare senior trainees for transition to the consultant role. We intend to run this course on a regular basis for interested senior trainees across the UK.

\section{G195 WHO'S THE LEAD? CAN A NOVEL TOOL AND TARGETED SIMULATION TRAINING IMPROVE PAEDIATRIC EMERGENCY LEADERSHIP?}

R Cotton, J Woodruff, M Anton, P Hernandez, M Lane, A Narayanan, L Casanueva, M Burmester. Paediatric Intensive Care, Royal Brompton Hospital, London, UK

\subsection{6/archdischild-2020-rcpch. 165}

Aims To prospectively measure the impact of the Leadership In Medical Emergencies (LIME) course through objective measurements of self and team perception of leadership and teamwork.

Methods 7 paediatric practitioners attended a one-day LIME course which introduced Crisis Resource Management and a novel LEAD-A-Team tool (table 1) followed by 8 simulated emergencies. Modified-Concise Assessment of Leadership Management (CALM) score was prospectively recorded by the leader and an observer. CALM (Nadkarni et al.) was modified to better reflect LEAD-A-Team, resulting in a maximum score 